\title{
Original \\ Asticle \\ Histological evaluation of extent of disease involvement in non-cirrhotic portal hypertension
}

\author{
Lavleen Singh ${ }^{1}$, Prasenjit Das ${ }^{1}$, Pratap Mouli², Rajni \\ Yadav $^{1}$, Ragini Kilambi ${ }^{3}$, Prateek Kinra ${ }^{1}$, Archana \\ George $^{1}$, Guresh Kumar ${ }^{4}$, Shalimar ${ }^{2}$, Subrat K Acharya ${ }^{2}$, \\ Siddhartha Datta Gupta ${ }^{1}$
}

Departments of ${ }^{1}$ Pathology,

${ }^{2}$ Gastroenterology, ${ }^{3}$ Gastrointestinal

Surgery and ${ }^{4}$ Biostatistics,

All India Institute of Medical Sciences,

New Delhi, India

Correspondence:

Siddhartha Datta Gupta

Email: sdattagupta@gmail.com

Prasenjit Das

Email: prasenaiims@gmail.com

\section{ABSTRACT}

Background : Non-cirrhotic portal fibrosis (NCPF) is a common cause of variceal bleed and can clinically mimic cirrhosis. Histological evaluation of a liver biopsy can serve multiple purposes.

Materials \& Methods: Retrieved liver biopsies from 41 patients of diagnosed NCPF and extra-hepatic portal vein obstruction (EHPVO) were included. The histopathological spectrum was compared. Light microscopic fibrosis staging in NCPF was compared with the fibroscan scores and liver fibrosis scores measured by computer assisted image analysis (CIA) technique. The extent of histological findings in portal tracts, and beyond the portal tracts was evaluated.

Results: While in NCPF, intra-hepatic portal vein thickening, thrombosis, obliterative portal venopathy (OPV), portal inflammation, peri-portal creeping fibrosis, peri-portal aberrant vascular channels were more frequent, nodular regenerative hyperplasia was common in EHPVO. In the former, fibrosis beyond portal tract was seen in almost one third of cases, however significant fibrosis was not seen in EHPVO. Liver fibrosis grades in NCPF did not correlate with elastogram values, but, correlated well with CIA measured fibrosis scores.

Conclusion: Histological evaluation in NCPF can give vital clues about diagnosis in an appropriate clinical setting, exclude cirrhosis and help in optimum fibrosis staging. In NCPF, the pathology is not restricted to portal tracts, but extends beyond them.

KEYWORDS: Non cirrhotic portal fibrosis; extra hepatic portal vein obstruction; obliterative portal venopathy; liver fibrosis staging; computer assisted image analysis; portal hypertension; fibroscan 


\section{Introduction}

Non-cirrhotic portal fibrosis (NCPF) is a clinicopathological condition characterized by fibrosis of the intra-hepatic portal veins (PV), resulting in development of portal hypertension, despite preserved liver function and patent spleno-portal axis. ${ }^{1}$ The basic etiology and pathogenesis still remains enigmatic, even after six decades of its first description in 1956 by a group of Indian scientists $^{2}$; though portal pyemia ${ }^{3}$, blood coagulation disorders, myeloproliferative diseases. ${ }^{4,5}$ exposure to toxic substances, drugs ${ }^{6}$ and immunological conditions ${ }^{7}$ have all been hypothetically linked to its pathogenesis. In India, $20-25 \%$ cases of well tolerated variceal bleed are attributed this condition. ${ }^{8}$ The pathological changes in NCPF are believed to be at the level of intra-hepatic presinusoidal PVs of all orders, including the large, mediumsized and pre-terminal branches $(0.2-3 \mathrm{~mm}$ in diameter $){ }^{9}$ With the advent of improved radiological tests, use of liver biopsy as the primary mode of diagnosis in NCPF has reduced. The recommendations on use of liver biopsy in NCPF also differ: while the European association of study of liver disease (EASL) recommends use of liver biopsy as an essential tool to exclude severe fibrosis or cirrhosis, ${ }^{10}$ the American association of study of liver disease (AASLD), does not recommend liver biopsy as an essential modality. ${ }^{11}$ The Asia-Pacific association of study of liver disease (APASL), on the other hand suggested a list of essential criteria including histological findings for diagnosis of NCPF. ${ }^{12}$ Liver biopsy often fails to serve its purpose in diagnosis of NCPF, as the cores obtained sample only one in fifty thousandth of liver parenchyma and may not show classical changes of obliterative portal venopathy (OPV) in the small PVs included. ${ }^{10}$ However, liver biopsy in NCPF especially becomes valuable where radiological features are not classical, and liver function tests are abnormal.

It was an important proposition to evaluate the histological changes of NCPF, in comparison to the extrahepatic portal vein obstruction (EHPVO), and to see how histological findings can help in a diagnosis, where the clinical presentation, or the radiological findings are not classical. It is also important to see if both of these portal venopathies are only limited to portal tracts, or there is extension of pathological findings beyond the portal tracts, which would be a major utility of taking a liver biopsy.

\section{Material and Method}

In this retrospective study, liver biopsies from 41 patients of clinically suspected, non-cirrhotic idiopathic portal hypertension (NCIPH) were included. Forty one cases of clinically suspected and radiologically confirmed EHPVO were also included as disease controls. Clinically a diagnosis of NCIPH was considered, when the following criteria were met in a given case: (i) evidence of portal hypertension (any one of the following features: esophageal varices, hyper-splenism, ascites, or increased hepatic venous pressure gradient); (ii) doppler ultrasound showing patent PV and hepatic veins (HV) at the time of diagnosis; (iii) preserved liver functions at presentation; (iv) exclusion of other conditions known to cause cirrhosis according to the conventional diagnostic criteria [chronic viral hepatitis, alcoholic liver disease, non-alcoholic steatohepatitis (NASH), obesity, hemochromatosis, autoimmune hepatitis, or Wilson's disease]. A transient elastogram (fibroscan) was performed in most of the cases for assessment of hepatic fibrosis. While tru-cut needle biopsies were available in all of them, wedge liver biopsy was also available in nine cases of EHPVO, in addition to the needle biopsies.

Formalin fixed and paraffin embedded (FFPE) sections were stained with Hematoxylin \& Eosin (H\&E), Masson's trichrome (MT), periodic acid Schiff (PAS), Gordon and Sweet's reticulin silver, and Sirius red (SR) stains. Multiple step sections of each liver biopsy core were examined and the number of portal tracts (PT) was counted in each of them for adequacy. The adequacy criteria considered for needle biopsy were the presence of at least 10 portal tracts, and a composite length of at least $1.5 \mathrm{~cm}$. However, a liver biopsy with at least 5 portal tracts, in presence of pathological findings, was also considered sufficient for interpretation. Biopsies were reviewed by the three pathologists (PD, SDG and LS) independently. A protocol of reviewing the biopsies was formulated including 27 histological criteria (Table 1), based on the available published data. These 
were: assessment of lobular architecture [Figure 1A], portal tract architecture/inflammation/interphase hepatitis/ hepatocyte ballooning/spotty necrosis, intrahepatic PV thickening/thrombosis/narrowing/obliteration/dilatation, portal and peri-portal fibrosis, dilated portal or peri-portal aberrant vessels, sinusoidal dilatation/inflammation, peri-sinusoidal fibrosis, Kupffer cell hypertrophy, hepatic vein (HV) dilatation/thickening/obliteration/ fibrosis, abnormal reticulin pattern, nodular regenerative hyperplasia (NRH), and presence of incomplete septal cirrhosis and atrophied or regressed portal tracts (RPT). Further semi-quantitative grading of the histological parameters were performed, where applicable [Table 1]. Nodular regeneration was defined as distinct nodularity $(<3 \mathrm{~mm})$ formed by hepatocytes, with peripheral rim of compressed atrophic hepatocytes, without any fibrosis, assessed on special stains. Incomplete septal cirrhosis was considered as thin fibrotic septa from portal tract ended short of the central vein. Abnormal reticulin pattern was considered as coarse parallely oriented reticulin fibers and loss of normal Chinese letter pattern of reticulin fiber arrangement around the hepatic sinusoids. Regressed portal tract (RPT) was defined as, atrophied tiny portal tracts with loss of vascular structures, often identified by only residual bile ducts.

Nakanuma, etal (1996) described four histological patterns on wedge biopsies from patients with idiopathic portal hypertension: normal histology, NRH, partial nodular transformation and incomplete septal cirrhosis. ${ }^{13}$ Following his descriptions, we though looked for these histological features; identification of partial nodular transformation of liver was out of scope as our study was based on tru-cut biopsies.

Portal vein was considered thickened if its lumen was compromised, and was further graded semi-quantitatively as mild, moderate and markedly thickened PV, by comparing it with accompanying hepatic artery. In physiological condition, the thickness of PV is less than the thickness of adjacent hepatic artery [Figure 1A].${ }^{14}$ The term mild obliterative portal venopathy was used when its lumen was compromised up to $25 \%$ of normal luminal diameter and its wall thickness is still less than the thickness of HA [Figure 1B]; moderate portal venopathy was used when luminal diameter of PV was compromised up to $50 \%$ in comparison to normal, or its wall thickness was equal to thickness of a HA [Figure 1C]; severe portal venopathy was used when PV lumen was compromised by $>50 \%$ or completely compromised, or PV wall thickness was more than thickness of HA [Figures 1D,1E]. Obliterative portal venopathy was characterized by completely obliterated $\mathrm{PV}$ with replacement by bland fibrosis (characterized by: loose collagen and lack of inflammatory cells), as identified by MT stain [Figures 1D,1E]. Changes in the central HV were also graded as mild, moderate and severe based on similar criteria of luminal compromise as described above for PV. The portal tract inflammation, spotty necrosis and piecemeal necrosis were graded histologically, by adopting the Ishak's modified histological activity index (HAI) grading system, used for chronic viral hepatitis. 15

The presence of these 27 histological criteria was compared between NCPF and EHPVO by using Chi square test, $\mathrm{p}$ value less than 0.05 was considered significant. Extent of hepatic fibrosis was assessed by Metavir staging as well as by computer assisted image analysis technique using Image Pro Plus 6.1 software (Image Pro, USA). Fibroscan values were assessed wherever available. Finally, the interpretations of all three observes were combined and compared.

Metavir fibrosis stages, CIA fibrosis values and fibroscan scores were compared by using Spearman's rank correlation coefficient test. The study was performed according to the Institutional ethical guidelines.

\section{Results}

\section{Patient profile}

The study comprised of 41 patients of clinico-radiologically confirmed NCPF, including 23 males and 18 females (M: F-1.2) with an average age of 27.2 years (range: 13 to 46 years). The age and sex matched liver biopsies of EHPVO were included as disease controls. On imaging, amongst the NCPF patients, 38 had splenomegaly (92.6\%). Twelve (29.2\%) of them presented with upper gastrointestinal bleeding and $11(91.6 \%)$ of these 12 patients had tolerated the bleed well, without any significant complication. 
Histological Features in liver biopsies in NCPF patients.

Most of the liver core biopsies showed only small pre-terminal branches of $\mathrm{PV}$, while the corresponding wedge biopsy specimens (n-9) also showed the presence of both medium and larger PV branches. While in 36 (87.8\%) out of the 41 cases of NCPF pathological features were identified, 9 biopsies (21.9\%) were within normal limits. The predominant histological findings in the former were: thickening and narrowing of PVs in $65.8 \%$ cases (33.3\% mild thickening and $66.7 \%$ moderate thickening), while OPV in $78.04 \%$ cases [Figures 1A-1E], including $\mathrm{PV}$ thrombus in 5 of the biopsies (12\%) [Figure 1E]. The next most common histological finding was aberrant vascular channels $(87.8 \%)$, identified at the interphase of the fibrous portal tracts and adjacent hepatocytes [Figure 1F, 1G]. These channels were irregular, thin walled, cleft like spaces without significant fibrosis and no muscle fibers in their wall. They were distinctly different from the portal vein, hepatic artery or the lobular hepatic sinusoids. The patent portal veins away from obliterative changes, in addition showed irregular dilatation and congestions (48.7\%). Chronic inflammatory cell infiltrate was noted in $70.7 \%$ of portal tracts. While mostly the inflammation was mild (55.2\%), moderate inflammation was also identified in $44.8 \%$ cases. None of the biopsies from NCPF showed severe inflammation [Table 1].

Disease extension was noted beyond the portal tracts in NCPF, as, in around $30-35 \%$ cases associated dilatation and thickening of the central vein (CV) was also noted. In only $2.4 \%$ cases CV obliteration was also seen, along with fibrosis around the CV (perisinusoidal fibrosis) in $34 \%$ of the biopsies of patients having NCPF [Figure 1H]. In most of the cases studied, the histological features described were present in combinations along with changes in the portal veins. Portal and peri-portal fibrosis was noted in $29 \%$ cases, which included pericellular creeping fibrosis around the hepatocytes in 24.2\% biopsies [Figure 1E]. Peri-sinusoidal creeping fibrosis was also noted at zone 1 in $12.2 \%$ of these cases. Sinusoidal dilatation (85.4\%) though common, was irregularly distributed, except only in $5.7 \%$ cases were sinusoidal dilatation was seen predominately in the peri-portal area [Figure 2A, Figure1]. Regressed portal tracts were seen in $58.5 \%$ cases, represented by very small absorbed portal tracts with obliterated/lost PV and/ or hepatic artery and an occasional residual bile duct. The stromal volume of the portal tract were markedly shrunken [Figure 2B]. Nodular regenerative hyperplasia (NRH) of hepatocytes was identified in $43.9 \%$ of cases [Figure 2C \& 2D], not surrounded by any fibrous bands. Rather, these nodules were encircled by atrophied, dark, small rounded hepatocytes [Figure 2C]. Reticulin stain showed focal formation of passive reculin condensation bands at the periphery of some of these nodules near the site of hepatocyte atrophy; the MT stain didn't reveal any collagenization [Figure 2D]. Most of the RPT were noted at the edges of regenerative nodules.

Though hepatocytes were mostly unremarkable in NCPF, focal ballooning was noted in $36.6 \%$ cases. In most of the index cases, Metavir stage 1 fibrosis (41.4\%) was seen, while stage 2 and 3 fibrosis were noted in $29.3 \%$ cases each. In our series, histopathological changes of portal venopathy were not only limited to the portal tract, but, there was also extension beyond the portal tracts in hepatic lobules, forming incomplete septal cirrhosis $(29.3 \%$ cases), where the thin fibrous septae from portal tracts were seen to abruptly end within the hepatic lobules [Figure 2E \& 2F; Table 1]. The transient elastography data was available in 31 of NCPF included in this study, where the liver stiffness scores ranged from 4.7 to 43.5 kilopascals with a mean value of 12.5 kilopascals, suggesting fibrosis within hepatic lobules in most of the cases. However, cirrhosis was not seen in any of the cases and there was no correlation between the Metavir fibrosis staging done on the liver biopsies and fibroscan values $(\mathrm{P}$ value $=0.7$, correlation coefficient $=$ 0.072 ). On the other hand, a positive correlation was found between the light microscopic Metavir staging and percentage fibrosis measured by the CIA technique ( $\mathrm{P}$ value $<0.001$, correlation coefficient $=0.73$ ) [Figure 3A \& 3B], supporting authenticity of our observations. 


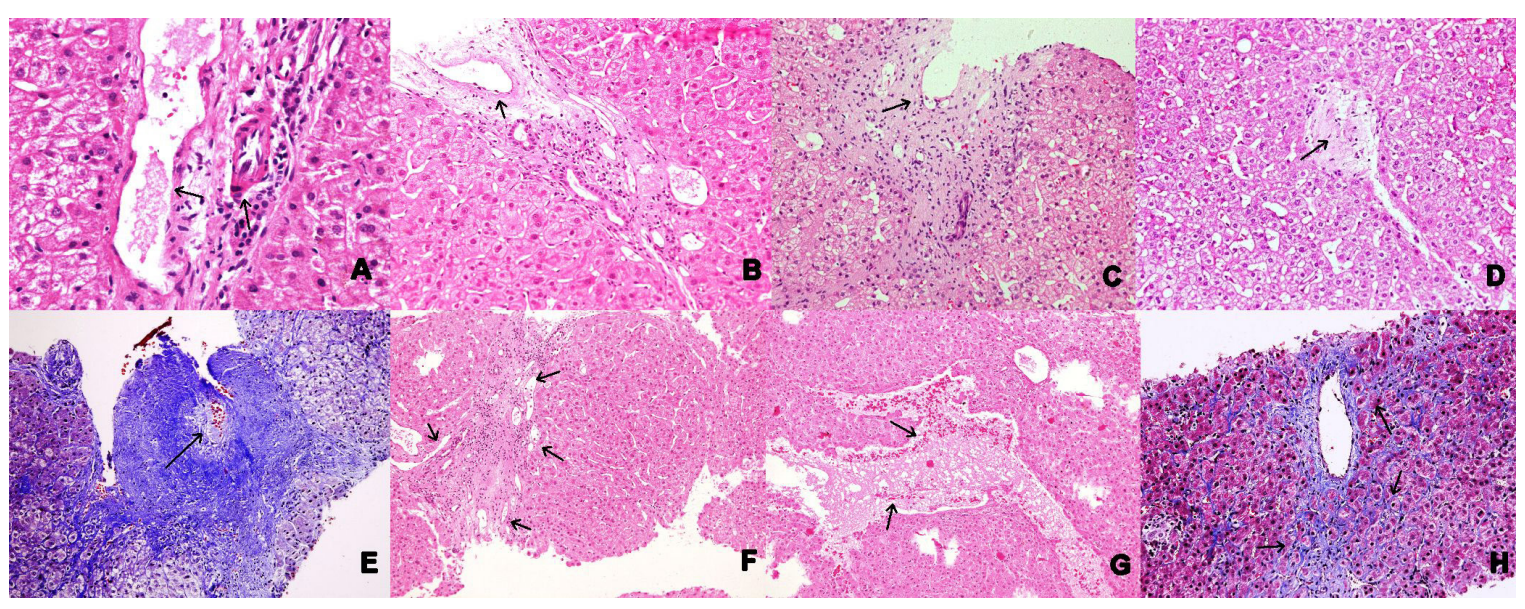

Figure 1: A. Method of identifying a normal portal vein (left arrow), in comparison to the thickness of adjacent hepatic artery (right arrow). Normal portal vein thickness is less than the thickness of hepatic artery [A, x200]. Biopsy showing portal venopathy and luminal compromise (arrow). Based on the thickness of PV and the extent of luminal compromise, portal venopathy changes histologically can be mild (arrow) [B x200], moderate (arrow) [C x200] and severe (arrow) [D x200]. A liver core-biopsy showing thrombosis of PV and severe obliterative portal venopathy (arrow) [E MT x200]. Another core biopsy showing severe obliterative portal venopathy with openedup peri-portal aberrant vascular channels (arrows) [F x200]. In occasional biopsies markedly dilated aberrant channels can protrude inside hepatic lobule, and appear as megasinusoids/ peliosis (arrows) [G x200]. Similar to PV changes, changes in hepatic vein can also be divided based on its wall thickness and extent of luminal compromise. In this figure mild luminal narrowing with peri-sinusoidal fibrosis (arrow) are seen [MT x 200].

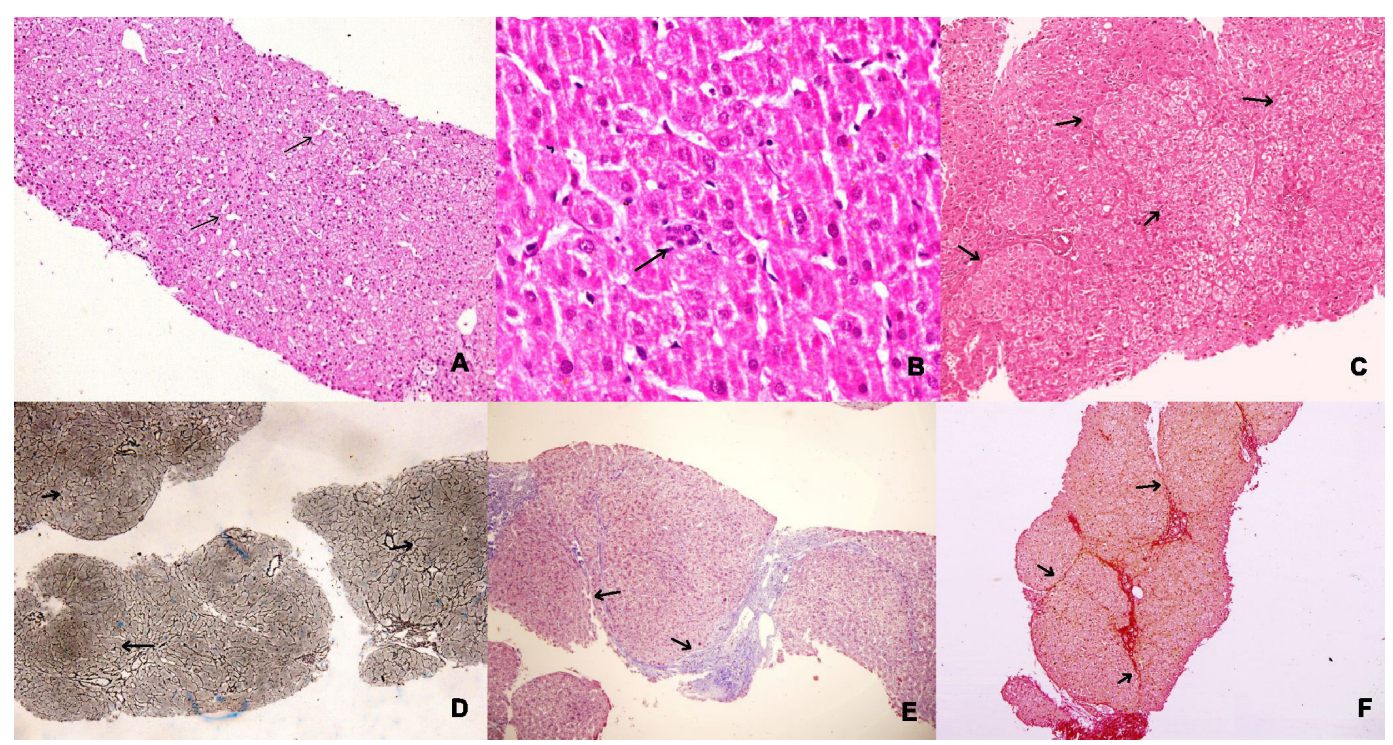

Figure 2: Photomicrograph showing a liver core biopsy from a case of NCPF, showing normal lobular architecture with irregular sinusoidal dilatation (arrows) [A x40]. Figure representing a small regressed portal tract with loss of portal collagen and residual bile duct (arrow) [B x400]. Core biopsy shows features of diffuse nodular regenerative hyperplasia. Varying size nodules (arrows) without surrounding fibrosis are seen [B x100]. Reticulin stain also shows nodular regenerative hyperplasia (arrow) with peripheral compressed reticulin network surrounding a regenerative nodule, without formation of septae [Reticulin stain $x$ 100]. Core biopsy showing incomplete septal cirrhosis, characterized by short thin fibrous septae extending from portal tract ending abruptly (arrows) within the hepatic lobule [E, MT x100; F, Sirius Red stain x 100]. 


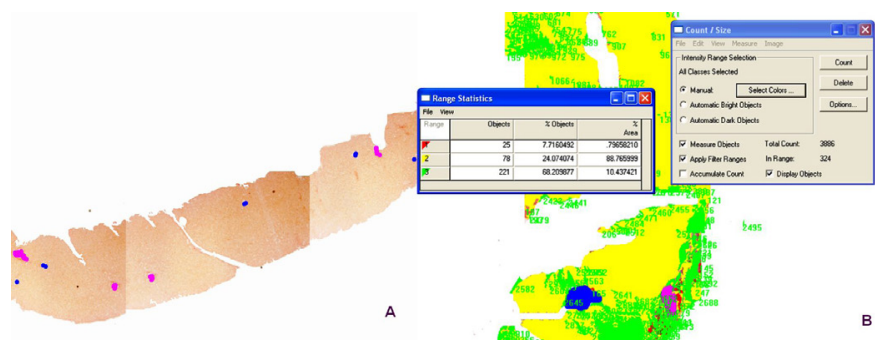

Figure 3: A composite core formed by serial tiling of digitally acquired images captured at $\times 40$ [A, Sirius red stain $x 40]$. A snap shot picture of the image analysis software screen showing method of color segmentation and measuring the fibrosis area [Fig B].

\section{Histological features in NCPF in comparison to the EHPVO}

Though PV dilatation was noted in both of these diseases, PV thickening ( $\mathrm{p}$ value $=0.04$ ) and OPV ( $\mathrm{p}$ value $<0.01$ ) were predominant findings in $\mathrm{NCPF}$ [Table 2]. Mild PV thickening was also noted in $43.9 \%$ of the EHPVO. Thrombosis of the intra-hepatic PV branches were noted in NCPF, however, were absent in EHPVO [Table 2]. Peri-portal aberrant vascular channels, were more commonly seen in NCPF cases as compared to EHPVO ( $\mathrm{p}$ value $=0.005$ ). The $\mathrm{CV}$ dilatation was also more frequent in NCPF, than in the cases of EHPVO (p value $=0.02)$. Sinusoidal dilatation $(p$ value $=0.04)$ and congestion ( $\mathrm{p}$ value $=0.03$ ) though were more frequent in cases of NCPF, were also noted in a subset of EHPVO. Megasinusoids, were however infrequent in both the conditions [Table 2]. RPTs were found to be a common finding in NCPF (58.5\%); they were found in only one case of EHPVO ( $p$ value $<0.01$ ). NRH was on the other hand commoner in EHPVO (61\%), than in the cases of NCPF $(43.9 \%)$. Portal inflammation up to moderate density was noted in two third patients with NCPF; in EHPVO portal inflammation was mild [Table 2]. Zone 1 creeping fibrosis was noted in a substantial proportion of NCPF cases $(70.7 \%)$, however similar fibrosis was negligible in EHPVO (p value $<0.01$ ) [Table 2].

\section{Discussion}

The present study was based on a group of patients clinically suspected to have NCIPH, with relative high fibroscan values in most, where the corresponding liver biopsies were evaluated. As both NCPF and EHPVO, involves the intra-hepatic and extra-hepatic branches of portal vein, it was an interesting proposition to compare the histological features of these conditions, as similar cases are not uncommon in routine clinical practice in this region. Clinically and radiologically often a NCPF appears as cirrhosis, ${ }^{16}$ or the clinical presentation may be atypical, where liver biopsy can contribute in clinical decision making. The diagnosis of EHPVO is on the other hand quite straightforward with aid of radiology.

We found that the presence of PV thickening, PV thrombosis, OPV, peri-portal aberrant vascular channels, peri-sinusoidal creeping fibrosis at zone 1, with regressed portal tracts are common findings in NCPF, which can aid in histological diagnosis in an appropriate clinical setting. Aberrant vascular channels may also be seen in EHPVO, though these channels in the latter were smaller and fewer in number. Portal inflammation and peri-portal fibrosis were also commoner in NCPF, than in EHPVO. Hepatic architecture in EHPVO was maintained in all cases included in this study. RPTs were commoner in NCPF, and if not examined under higher magnification, these tiny regressed structures may be missed. Sinusoidal dilatation or dilatation of hepatic and portal veins were found to be nonspecific findings and can be seen in both of these conditions [Table 2]. Megasinusoids, i.e. presence of large dilated sinusoid with disruption of surrounding reticulin architecture were relatively rare in both the diseases, and are regarded as protrusion of the dilated aberrant channels into the hepatic lobules.

In comparison to all histological features described, nodular regenerative hyperplasia on the other hand was commoner in EHPVO (61\%), than in NCPF (43.9\%). Nakayuama et al. identified NRH frequently in NCPF. ${ }^{17}$ These regenerative nodules are possibly formed due to hypertrophy of the hepatocyte acini receiving blood supply from a patent PV branch, while the obliterated vein branches result in atrophy of hepatocytes and regressed portal tracts at the zone of its supply. But as the hepatic artery is still preserved in the zone of hypoperfusion, ischemic necrosis possibly does not set in. The RPTs were also identified by Verheij et al and Wanless et al, in NCPF and was thought to be formed due to ischemic regression 
Table 1: Histopathological features and their frequency in liver biopsies of NCPF cases

\begin{tabular}{|c|c|c|}
\hline Histopathological parameter & Frequency $(n-41)$ & Percentage $(\%)$ \\
\hline $\begin{array}{l}\text { Portal vein Thickening } \\
\text { Mild } \\
\text { Moderate }\end{array}$ & $\begin{array}{l}27 \\
9 \\
18\end{array}$ & $\begin{array}{l}65.8 \\
33.3 \\
66.7\end{array}$ \\
\hline Obliterative portal venopathy & 32 & 78 \\
\hline Portal vein thrombus & 5 & 12.2 \\
\hline Portal vein dilation & 20 & 48.7 \\
\hline Peri-portal aberrant vascular channels & 36 & 87.8 \\
\hline Regressed portal tracts & 24 & 58.5 \\
\hline $\begin{array}{l}\text { Sinusoidal dilatation } \\
\text { Zone } 3 \\
\text { Diffuse } \\
\text { Irregular }\end{array}$ & $\begin{array}{l}35 \\
2 \\
6 \\
27\end{array}$ & $\begin{array}{l}85.4 \\
5.7 \\
17.1 \\
77.1\end{array}$ \\
\hline Dilation of the central veins & 14 & 34.1 \\
\hline Thickening of central vein & 13 & 31.7 \\
\hline Central vein obliteration & 1 & 2.43 \\
\hline Central vein fibrosis & 14 & 34.1 \\
\hline Portal and peri-portal fibrosis & 12 & 29.3 \\
\hline Peri-sinusoidal creeping fibrosis & 5 & 12.2 \\
\hline Peri-cellular fibrosis & 10 & 24.2 \\
\hline $\begin{array}{l}\text { Portal inflammation } \\
\text { Mild } \\
\text { Moderate } \\
\text { Severe }\end{array}$ & $\begin{array}{l}29 \\
16 \\
13 \\
0\end{array}$ & \begin{tabular}{|l|}
70.7 \\
55.2 \\
44.8 \\
0
\end{tabular} \\
\hline Sinusoidal inflammation & 5 & 12.2 \\
\hline Nodular regenerative hyperplasia & 18 & 43.9 \\
\hline $\begin{array}{r}\text { Spotty necrosis } \\
<2 / 10 \mathrm{HPF} \\
>2 / 10 \mathrm{HPF}\end{array}$ & $\begin{array}{l}19 \\
19 \\
0\end{array}$ & $\begin{array}{l}46.3 \\
46.3 \\
0\end{array}$ \\
\hline $\begin{array}{l}\text { Piecemeal necrosis } \\
\text { Focal, in some portal tracts } \\
\text { Moderate, in all portal tracts }\end{array}$ & $\begin{array}{l}10 \\
10 \\
0\end{array}$ & $\begin{array}{l}24.4 \\
24.4 \\
0\end{array}$ \\
\hline Hepatocyte ballooning & 12 & 29.3 \\
\hline Ballooning of hepatocytes & 15 & 36.6 \\
\hline Kupffer cell hypertrophy & 9 & 21.9 \\
\hline Abnormal reticulin pattern & 21 & 51.2 \\
\hline $\begin{array}{l}\text { Metavir Stage } \\
\text { Stage } 1 \\
\text { Stage } 2 \\
\text { Stage } 3 \text { [Incomplete septal cirrhosis] }\end{array}$ & $\begin{array}{l}17 \\
12 \\
12\end{array}$ & $\begin{array}{l}41.4 \\
29.3 \\
29.3\end{array}$ \\
\hline
\end{tabular}


Table 2: Comparison of histological findings between NCPF and EHPVO

\begin{tabular}{|c|c|c|c|}
\hline Histopathological Parameters & $\operatorname{NCPF}(\mathrm{N}=41)[\mathrm{n}(\%)]$ & EHPVO $(\mathrm{N}=41)[\mathrm{n}(\%)]$ & p-value \\
\hline Portal vein thickening & $27(65.8)$ & $18(43.9)$ & 0.04 \\
\hline Obliterative portal venopathy & $29(70.7)$ & $1(2.4)$ & $<0.01$ \\
\hline Intra-hepatic PV thrombosis & $5(12.2)$ & $0(0)$ & 0.08 \\
\hline Dilated portal vein & $20(48.78)$ & $22(53.7)$ & 0.6 \\
\hline Peri-portal aberrant vessels & $36(87.8)$ & $25(61)$ & 0.005 \\
\hline Dilated central vein & $14(34.14)$ & $3(7.31)$ & 0.02 \\
\hline $\begin{array}{l}\text { Sinusoidal dilatation } \\
\text { Zone } 3 \\
\text { Diffuse } \\
\text { Irregular }\end{array}$ & $\begin{array}{l}35(85.4) \\
2(5.7) \\
6(17.14) \\
27(77.14)\end{array}$ & $\begin{array}{l}10(24.4) \\
0(0) \\
2(20) \\
8(80)\end{array}$ & 0.04 \\
\hline Sinusoidal congestion & $19(46.3)$ & $10(24.4)$ & 0.03 \\
\hline Mega-sinusoids & $2(4.8)$ & & \\
\hline Peri-portal & $1(2.4)$ & 0.6 & \\
\hline Regressed portal tracts & $24(58.5)$ & $1(2.4)$ & $<0.01$ \\
\hline $\begin{array}{l}\text { Nodular regenerative hyperpla- } \\
\text { sia (NRH) }\end{array}$ & $18(43.9)$ & $25(61)$ & 0.12 \\
\hline $\begin{array}{l}\text { Portal inflammation } \\
\text { Mild } \\
\text { Moderate }\end{array}$ & $\begin{array}{l}29(70.7) \\
16(55.2) \\
13(44.82)\end{array}$ & $\begin{array}{l}28(68.3) \\
26(92.9) \\
2(7.1)\end{array}$ & 0.81 \\
\hline Zone 1 creeping fibrosis & $29(70.7)$ & $1(2.4)$ & $<0.01$ \\
\hline Zone 3 creeping fibrosis & $0(0)$ & $0(0)$ & - \\
\hline $\begin{array}{l}\text { Architectural distortion with } \\
\text { incomplete nodule formation\# }\end{array}$ & $12(29.3)$ & $0(0)$ & $<0.01$ \\
\hline
\end{tabular}

\# Equivalent to the incomplete septal cirrhosis in NCPF. 
of portal collagen. ${ }^{18,19}$ In EHPVO also alteration of hepatic blood flow might be responsible for formation of these nodules. However, central HV dilatation and thickening were not uncommon in NCPF (noted in $73 \%$ cases). This may either be due to increased hepatic venous-wedge pressure or due to the effects of circulating toxin on $\mathrm{HV}$ endothelial cells. ${ }^{20} \mathrm{HV}$ fibrosis $(31.7 \%)$ and luminal obliteration of HV were rare in NCPF [Table 2]. Many authors have put forth hypothesis of circulating fibrogenic toxins in the portal circulation as the probable cause of PV fibrosis and luminal obliteration in NCPF. The degree and percentage of the changes in HV may be less than the $\mathrm{PV}$, due to the neutralizing effects of these toxins during transit from $\mathrm{PV}$ to $\mathrm{HV}^{20}$

In contrast to the previous studies on chronic hepatitis, no correlation was identified between the extent of liver fibrosis, estimated by light microscopic examination and non-invasive elastogram fibrosis scores. ${ }^{21,22}$ Moreover, in the index cases mean elastogram score was quite high, giving a clinical suspicion of cirrhosis. The elastogram scores may be influenced by frequent presence of changes like $\mathrm{NRH}$, sinusoidal fibrosis, OPV and incomplete septal cirrhosis etc., which may lead to an erroneous stiffness score. On the other hand we have seen that light microscopic examination in the index cases ruled out cirrhosis in all, showed presence of mild to moderate portal and peri-portal fibrosis in some and presence of incomplete fibrous septae in around $12 \%-13 \%$ of the cases. Liver biopsy in NCPF, hence, not only can confirm the diagnosis, it can help in accurate staging, with the limitation of area of sampling. Our observations on light microscopy were also correlated with CIA fibrosis scores, suggesting that liver biopsy in NCPF can be valuable for fibrosis assessment. CIA technique is increasingly being used nowadays and is considered more objective. ${ }^{23}$ However, a word of caution is that, like any other technique, proper knowledge of calibration, suitability of using various processing filters and optimal segmentation procedures need to be mastered before one can use CIA technique in routine practice. Image processing, was also a vital step in image analysis, as one has to learn how to subtract the noisy background, so that they don't interfere while selecting the areas with similar pixel values. Incomplete septal cirrhosis, which was also identified exclusively in cases of NCPF, may represent the hepatic repair complex, or, may represent an advance stage of the disease, as hepatic fibrosis is a dynamic process, depending on the alterations of portal hemodynamics. ${ }^{24}$ But, looking at all the features, it is apparent that pathological features in NCPF are not limited to portal tracts; which may interfere with the non-invasive fibrosis assessment, resulting in erroneous clinical impression. ${ }^{16}$ It appears that, most of the recommendations by hepatology associations on utility of liver biopsy in vascular liver diseases, as NCPF, are correct and a liver biopsy may be recommended, in any clinical scenario, where liver architecture appears coarse, but biochemical findings are not supportive. It is important to identify NCPF, especially to differentiate it from cirrhosis, as in the former overall prognosis is good, possibly due to relatively preserved hepatocyte function; though, both of these can present with significant variceal bleed. A timely diagnosis and assessment of extent of chronicity may help in proper clinical decision making.

\section{Conclusion}

NCIPH is a group of clinico-pathological entities, where liver biopsy should be performed to rule out cirrhosis, differentiate between NCPF and EHPVO, with the support of adequate clinical information and radiological detail. Liver biopsy may also help to stage the extent of fibrosis optimally. Noninvasive elastogram, though convenient, may not be accurate in assessing liver fibrosis in this scenario. In NCPF, the histological findings are not only limited to the portal tracts, but also extends beyond it into the hepatic lobule, whereas, EHPVO is mostly limited to the portal perimeter.

\section{References}

1. Sharma P, Kumar A, Mehta V, Sharma BC, Sarin SK. Systemic and pulmonary hemodynamics in patients with non-cirrhotic portal fibrosis (NCPF) is similar to compensated cirrhosis. Hepatol Int. 2007;1(1):275-80.

2. Ramalingaswamy B, Wig KL, Sama SK. Cirrhosis of the liver in northern India. A clinicopathological study. Arch. Intern. Med.1962; 110: 350-8. 
3. Sarin SK. Non-cirrhotic portal fibrosis. J Gastroenterol \& Hepatol 2002;17:S214-S223

4. Wanless IR, Godwin TA, Allen F, Feder A. Nodular regenerative hyperplasia of the liver in hematologic disorders: A possible response to obliterative portal venopathy. A morphometric study of nine cases with a hypothesis on the pathogenesis. Medicine (Baltimore) 1980;59:367-79

5. Wanless IR, Peterson P, Das A, Boitnott JK, Moore GW, Bernier V. Hepatic vascular disease and portal hypertension in polycythemia vera and angiogenic myeloid metaplasia: a clinicopathological study of 145 patients examined at autopsy. J Hepatol 1990;12:1166-74.

6. Solis-Herruzo JA, Vidal JV, Colina F, Santalla F, Castellano G. Nodular regenerative hyperplasia of the liver associated with the toxic oil syndrome: report of five cases. J Hepatol 1986;6:687-69

7. Sekiya M, Sekigawa I, Hishikawa T, Iida N, Hashimoto $\mathrm{H}$, Hirose $\mathrm{S}$. Nodular regenerative hyperplasia of the liver in systemic lupus erythematosus. The relationship with anticardiolipin antibody and lupus anticoagulant. Scand J Rheumatol 1997;26:215-17.

8. Sarin SK, Aggarwal SR. Idiopathic portal hypertension. Digestion 1998;59:420-23.

9. Cazals-Hatem D, Hillaire S, Rudler M, Plessier A, Paradis V, Condat B, et al. Obliterative portal venopathy: Portal hypertension is not always present at diagnosis. J Hepatol. 2011;54(3):455-61.

10. EASL Clinical Practice Guidelines: Vascular diseases of the liver. J Hepatol (2015), http://dx.doi.org/10.1016/j. jhep.2015.07.040

11. Rockey DC, Caldwell SH, Goodman ZD, Nelson RC, Smith AD. Liver Biopsy. HEPATOLOGY. 2009;1:17-44.

12. Sarin SK, Kumar A, Chawla YK, Baijal SS, Dhiman RK, Jafri W, et al; Members of the APASL Working Party on Portal Hypertension. Noncirrhotic portal fibrosis/ idiopathic portal hypertension: APASL recommendations for diagnosis and treatment. Hepatol Int. 2007;1(3):398413.

13. Nakanuma Y, Hoso M, Sasaki M, Terada T, Katayanagi $\mathrm{K}$, Nonomura A, et al. Histopathology of the liver in non-cirrhotic portal hypertension of unknown aetiology. Histopathology. 1996;28(3):195-204.
14. H€ubscher SG. Pathology of non-cirrhotic portal hypertension and incomplete incomplete septal cirrhosis. Diag Histopathol. 2011;17(12):530.

15. Brunt EM. Grading and staging the histopathological lesions of chronic hepatitis: the Knodell histology activity index and beyond. Hepatology. 2000;31(1):241-46.

16. Nayak NC, Jain D, Saigal S, Soin AS. Non-cirrhotic portal fibrosis: one disease with many names? An analysis from morphological study of native explant livers with end stage chronic liver disease. J ClinPathol. 2011;64(7):592-98.

17. Nakanuma Y, Tsuneyama K, Ohbu M, Katayanagi K. Pathology and pathogenesis of idiopathic portal hypertension with an emphasis on the liver. Pathol Res Pract.2001;197(2):65-76.

18. Wanless IR, Nakashima E, Sherman M. Regression of human cirrhosis. Morphologic features and the genesis of incomplete septal cirrhosis. Arch Pathol Lab Med. 2000;124:1599-607.

19. Verheij J, Schouten JNL, Komuta M, Nevens F, Hansen BE, Janssen HLA, et al. Histological features in western patients with idiopathic non-cirrhotic portal hypertension. Histopathology. 2013;62:1083-91.

20. Schouten JNL, Verheij J, Seijo S. Idiopathic non-cirrhotic portal hypertension: a review. Schouten et al. Orphanet Journal of Rare Diseases 2015;10:67.

21. Castéra L, Vergniol J, Foucher J, Le Bail B, Chanteloup E, Haaser M, et al. Prospective comparison of transient elastography, Fibrotest, APRI, and liver biopsy for the assessment of fibrosis in chronic hepatitis C. Gastroenterol. 2005;128(2):343-50.

22. Goyal R, Mallick SR, Mahanta M, Kedia S, Shalimar, Dhingra R, et al. Fibroscan can avoid liver biopsy in Indian patients with chronic hepatitis B. J Gastroenterol Hepatol. 2013;28(11):1738-45.

23. Huang Y, de Boer WB, Adams LA, MacQuillan G, Bulsara MK, Jeffrey GP. Image analysis of liver biopsy samples measures fibrosis and predicts clinical outcome. J Hepatol. 2014;61(1):22-7.

24. Zenali M, Lidofsky SD. Beyond Degree of FibrosisAssessment of Liver Biopsy when there is Clinical Concern for Portal Hypertension. Ann Clin Pathol 2013;1(1):1004. 